\title{
The mini and micro as intelligent terminals
}

\author{
JONATHAN VAUGHAN \\ Hamilton College, Clinton, New York 13323
}

\begin{abstract}
A PDP. 8 has been linked by the telephone to an IBM 370 using the conventional OS/8 and CMS operating systems. Data acquired in on-line experiments are transferred, and analysis programs and batch jobs are submitted, directly from the PDP-8 disk to CMS. A subset of the system is used on an Intercept Jr. microcomputer when the PDP-8 is not free.
\end{abstract}

Many experimental psychologists now use both dedicated minicomputers in their laboratories and larger interactive timesharing systems on a department or university level. Each of these systems performs a particular kind of computing task better than the other. The laboratory minicomputer excels at the acquisition of data and control of on-line experiments, but it is relatively inefficient at handling large amounts of data in higher level programming languages or when complex statistical analysis packages are required. The university computer center excels at these, but it cannot easily be used for the acquisition of on-line data.

By historical "accident," there is no generally available, inexpensive and reliable medium for the transfer of information between laboratory minicomputers (most often, Digital Equipment Corporation machines) and the larger machines (typically, IBM) in university computer centers. Reduction of large amounts of data on the mini is limited in many laboratories by small available memory, slow floating-point routines, higher level languages with inconvenient diagnostics, only six-digit accuracy, or nonexistent statistical packages. ${ }^{1}$ Printing data, then manually keypunching it for entry into the larger machine introduces a new set of problems, including slow turn-around time, cost, and possible inaccuracy.

One way to take advantage of the best features of each type of computer system is to interface the two. This can be done economically at 30 characters/sec using the dial-up telephone network and an inexpensive acoustic coupler, or modem. This approach is slow, but provides an accurate and automatic way in which to transfer small to moderately large amounts of data. Thus, a modest computer network is created, with the real-time response requirements assigned to the laboratory mini and the less time-critical computational load delegated to the computer center. While the link between the mini and computer center has

A number of people have given assistance and/or technical advice in the development of this project. They are (alphabetically) Dan Bartholemew, Tom Brackett, Bob Hyman, Jim Manning, Dave Smallen, and Joan Winters. This work was supported by Grant MH 26303 from the National Institute of Mental Health. been established before (e.g., Hiltz, 1975), details of the method are not generally available to psychologists.

Such a system has been implemented between the PDP-8 minicomputer in the psychology department at Hamilton College and the IBM 370 at Cornell University, in such a way as to maintain compatibility with OS/8 (operating system for PDP-8) and CMS (conversational monitor system), that are most often used on the two machines. This link was designed with the following two constraints in mind. First, it should rely as much as possible on the standard use of the operating systems (file formats, character codes, and so forth) that are available for the two machines involved. While this limits flexibility somewhat, it is more than repaid in time saved by not having to work with idiosyncratic data formats or unfamiliar assembly-language routines. In addition, it increases the ultimate portability of the system developed for application to other laboratories or other manufacturer's computer systems. Second, the direction of data transfer has been heretofore limited, from the mini to the computer center, because the author's primary concern has been the analysis of large amounts of data generated by the mini, using statistical packages available only on the larger machine. Similar applications (Grupsmith, 1978; Hiltz, 1975; Lemkin, Note 1) have taken advantage of two-way data transfers. ${ }^{2}$

\section{PRINCIPLES OF OPERATION}

In the laboratory, on-line data are acquired and analysis programs and batch jobs are prepared as OS/8 files for later submission to CMS. The connection over the dial-up telephone link between the two machines can be limited, then, to the time necessary to transmit files to CMS from the PDP-8 disk, which is done in alphanumeric (ASCII) format compatible with both systems. All information transfer is buffered to make optimum use of the telephone link. Operations involving the link can be interleaved with other OS/8 operations, such as editing files, without disconnecting the link. This has proved to be a particularly appreciated feature. The TECO text editing program for the PDP-8, especially when enhanced with continuous CRT display 
of the text buffer, is more versatile than the CMS editor, as well as more familiar to the author. Thus, it is more convenient to prepare program and control files in the normal OS/8 environment for subsequent transmission to CMS than to use the CMS system in conventional fashion.

Once data have been transferred from the PDP- 8 to the 370 , they may be accessed as any other file in the on-line disk system, from the CMS timesharing system or the HASP batch entry system.

\section{SYSTEM CHARACTERISTICS}

\section{Hardware}

The system elements used in this application are shown in Figure 1. The basic approach is to make the PDP-8 appear to the IBM 370 as one of its many timesharing terminals, presumably under manual control; so, let us first look at the characteristics of that application. In general timesharing use, input to CMS is handled through an IBM 3705 communications controller front end, which accepts input from remote terminals over the dial-up line at 110 or 300 baud ( 10 or 30 characters $/ \mathrm{sec}$ ) in either the ASCII or EBCDIC character code set. While the connection to the 3705 is electronically a full duplex modem port, CMS treats input as if it were half duplex. That is, the remote timesharing terminal must be treated as being in one or the other of two mutually exclusive modes, input or output, at any particular moment. During the input mode, a line of input may be typed in by the user. Detection of the end-of-line character (ASCII 2158) by the 3705 switches it to output mode when characters are sent to the remote terminal and no input is accepted. When ready for more input, CMS transmits a variable number of characters, including the "line feed" and "X-ON" (ASCII $21_{8}$ ) characters. (This is detected by some timesharing terminals as a command to unlock the keyboard or start a paper-tape reader.)

Hardware was developed to allow the PDP- 8 to communicate with the dial-up telephone line in a manner similar to commercially available timesharing terminals such as a standard teletypewriter. A 300-baud universal asynchronous receiver-transmitter (UART, Number 6402, Intersil, Inc., Tantau, California) was implemented using a 26-DE-8 parallel $1 / 0$ transfer breadboard plugged into the PDP-8/E omnibus (Douglas Electronics, San Leandro, California). The UART was connected by an optically isolated 20 -mA current loop to a standard acoustic coupler (FM300, Multitech Systems, Inc., Minneapolis, Minnesota). ${ }^{3}$ Ordinarily, the 3705 uses the "break" function of the standard Teletype as an indication that output from CMS should be interrupted. This function was simulated on the I/O transfer board using a spare IOT instruction to cause the $20-\mathrm{mA}$ loop to be interrupted for $300 \mathrm{msec}$ when a control key ("control-D") is struck on the console Teletype. ${ }^{4}$

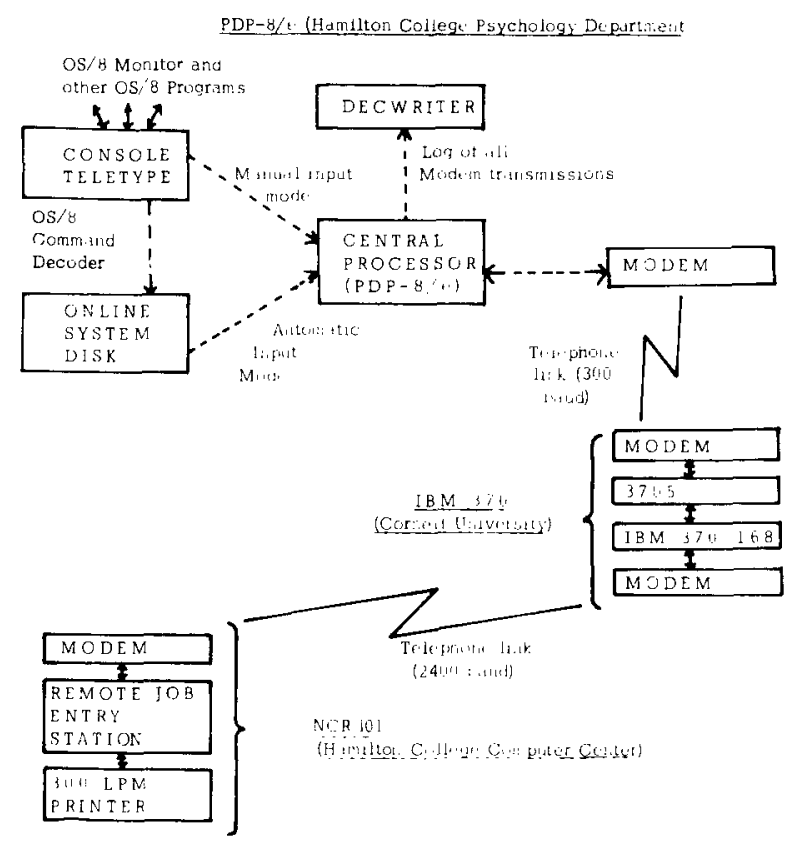

Figure 1. The system by which the $\mathrm{PDP}-8 / \mathrm{E}$ is made to appear to the IBM 3705 communications controller as a timesharing terminal. The telephone link between the PDP-8 and the 3705 is by the dial-up system; the link between the IBM 370 and the NCR101 at Hamilton College is a leased line.

\section{Software}

A utility program was written in PAL 8 assembly code for the PDP- 8 to accept character input from the keyboard or from the PDP- 8 disk. Input is sent over the phone line to the 3705 ; at the same time, input and any output from the 3705 are echoed on a receive-only DECwriter. After each input line has been sent to the 3705, the program waits until receipt of the line is acknowledged by CMS with the ASCII X-ON character. When this character has been received by the PDP-8, the next line of input is transmitted. A detailed exposition of the line protocol of CMS, and how program outputs may be synchronized with it, is presented elsewhere in these Proceedings (Grupsmith, 1978).

The source of input to be transmitted depends on the input mode, which may be manual or automatic. In the manual mode, characters are transmitted to CMS just as they are typed into the console Teletype keyboard. Teletype input is buffered so that the line protocol of CMS is invisible to the user.

To select automatic input mode, the operator types a reserved control character ("control-B"). First, the OS/8 command decoder is called, and the names of the files to be transmitted from the PDP- 8 are typed in by the operator. Up to nine files may be concatenated. The contents of these files, which must be ASCII alphanumeric information, are then automatically transmitted to CMS. The nature of the file transferred is otherwise arbitrary: It may be data, a program to be run under CMS or batch at the 370 , or it may be a series of direct 
commands to the CMS system, such as file manipulation, or batch job submission. Long data files are accompanied by a check-sum for each line, the accuracy of which is later verified once the data file is resident on a 370 systems disk. The error rate is low: some 10 errors (dropped characters or garbled lines) in about twomillion characters transferred. All errors are detected by either incorrect line counts in transferred data files or check-sum irregularities during verification by a FORTRAN program.

All input and output are suitably buffered so as to maintain transmission and reception of information at a rate that is alternately limited by the $30-\operatorname{cps}(300$. baud) modem connection in input mode and by the response time of the timesharing system in output mode. The resulting average transmission rate for automatic input is between 15 and $20 \mathrm{cps}$, depending on the timesharing system's response time.

\section{APPLICATIONS}

\section{A Timesharing Terminal}

In manual input mode, the console Teletype of the PDP-8 serves as an ASCII timesharing terminal, with output appearing on the DECwriter at $30 \mathrm{cps}$.

\section{Batch Job Submission}

Jobs may be submitted to the HASP batch system in manual input mode or directly from previously prepared files on the PDP-8 disk. Batch output may be directed to the PDP-8 (appearing on the DECwriter) or to the remote job entry station in the Hamilton College Computer Center, where it may be listed on a 300-lpm printer.

\section{Transfer of Data}

Data files may be transferred to CMS or to the HASP card-file system files on the $\mathbf{3 7 0}$ automatically from the PDP-8 disk. A file representing the data of one subject in a visual search experiment, suitably compressed, represents some 110 blocks on the OS/8 system device, or 40,000 characters of alphanumeric information. Such a file requires $30-40$ min to transmit to a "card file" system file.

\section{EXAMPLE}

An example of the use of the system to transmit one data file onto the IBM 370 system disk is shown in Figure 2. The Teletype console and DECwriter output are from a sample "terminal session" transferring a file of data from the PDP- 8 disk to a card file on the IBM 370 system. Note that there is an (intentional) error in the header file and how this is corrected using the TECO editor in the normal OS/8 environment.

A. HEAD.DA is an ASCII file on the PDP-8 disk that contains the commands necessary for the CMS operating system to submit a job (named "FILE") to create a file (named "DATA") under a HASP user's account (account XFQ). The output of this HASP job is to be delivered to the RJE station at the Hamilton College Computer Center ("DLVR HAM"). Note that there is a typographical error: The account number is misspelled "XFQZ," which is illegal, having four rather than three characters. (The format of commands necessary to accomplish this task may differ at other installations.) The file is typed on the console teletypewriter by the OS/8 "TYPE" command.

B. DATA.DA is an ASCII data file such as might have been created by an on-line experiment. Each line comprises three numbers in FORTRAN I3 format, with a fourth number, the check-sum, being the sum of the first three. The check-sum permits later verification of correct transmission.

C. END.DA is a file containing the CMS commands necessary to terminate the HASP input.

D. At this point, the dial-up telephone link has been established with the acoustic coupler, the communications program (CORN.SV) is called, and the console Teletype used to begin a timesharing session under the appropriate access code (KCF). The input from the console Teletype is echoed on the DECwriter as it is forwarded to the 370; the 15-line message of the day from the timesharing system is then received.

E. The automatic mode is selected using the control-B key (nonprinting). Three input files are specified for the OS/8 command decoder, and transmission of the files to the timesharing system is begun. The operator notices the error, interrupts the automatic mode by the control-D (simulated "break") key, and returns to OS/8 via control-C.

F. Using the TECO editor, the offending line is typed out $\left(\mathrm{F}_{1}\right)$, corrected using the TECO "FS" function $\left(F_{2}\right)$, and verified as correct $\left(F_{3}\right)$.

G. The communications program (CORN.SV) is again called. Note that the telephone link has been maintained, and it is not necessary to log on again. The timesharing session is reentered just where it was left.

$H$. The automatic mode is again selected using the control-B key, and the three input files again specified for the OS $/ 8$ command decoder. This time they are transmitted without a hitch. Note the interleaving of the lines of the input files with the responses of the timesharing system. The HASP batch job submitted will be treated as any other job submitted.

I. At the conclusion of transmission of the files and reception of the system response, the communications program returns to the manual input mode. The operator logs off the timesharing system and manually disconnects the telephone link by hanging up. Note that this session cost 16 cents (at an evening discount) in timesharing system charges, including CPU and connect time. 


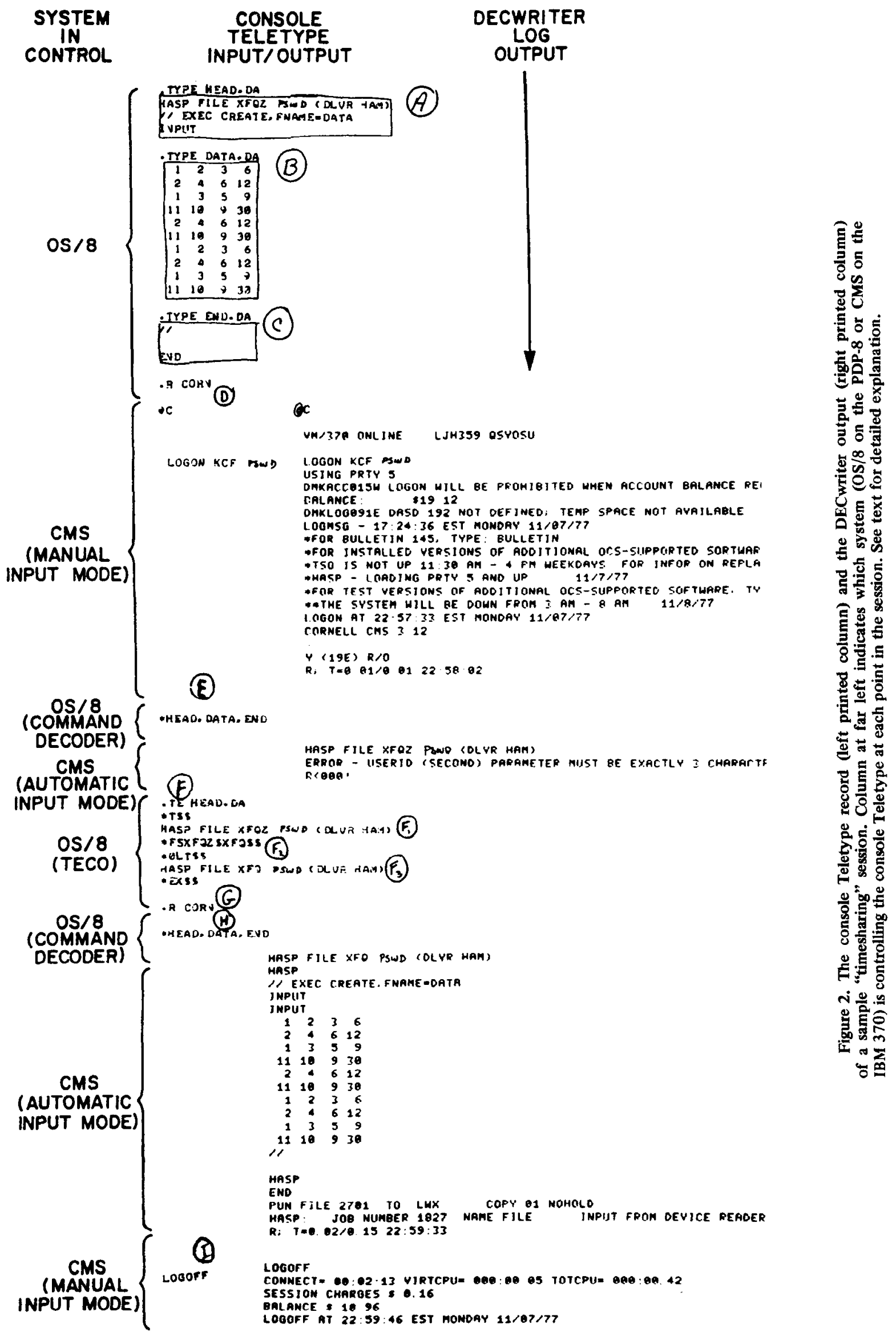




\section{THE MICRO}

A subset of the system described here has been implemented using the Intercept Jr. single-board microcomputer (Intersil, Inc., Tantau, California). This microcomputer was selected because its instruction set is identical to that of the PDP-8. So far this has been used simply as a remote timesharing terminal when the PDP-8 is not available for this use. The Intercept Jr. is a 256-word-memory one-board microcomputer. Expansion memory to $3 \mathrm{~K}$ words, serial UART interface, and a 12 -bit parallel $\mathrm{I} / \mathrm{O}$ board are available. ${ }^{5}$ Two of the serial $\mathrm{I} / \mathrm{O}$ boards are used in the present application, one for connection to the modem and the other for connection to a Teletype.

The availability of an easily interfaced parallel $\mathrm{I} / \mathrm{O}$ board for the Intercept Jr. suggests the potential for the application of remote timesharing systems to laboratory situations which require input and output of digital data. It is well within the capabilities of the Intercept Jr. with $1 \mathrm{~K}$ of memory to control a trial of a moderately complex procedure, then (in the intertrial interval) forward to the host timesharing system summary data from that trial, as long as the response time of the timesharing system does not exceed the intertrial interval. This is one simple way to distribute on-line control and computation in a computer "network."

We are also developing a system based on the Intercept Jr. to record on punched paper tape the reaction times from memory experiments conducted on a three-channel tachistoscope. The data will be read by the PDP-8 high-speed paper-tape reader and transmitted to the IBM 370 in the usual way.

\section{CONCLUSIONS}

The system described here has proved to be a useful and economical approach to interprocessor communications for the laboratory computer user. Though it is slow, the system allows the analysis of large amounts of data and the use of sophisticated statistical packages that are beyond the capacity of most laboratory minicomputers. In particular, data can be transferred from one machine to the other much more quickly and accurately than the only alternative realistically available to the user-manual card punching.

With this system, it is theoretically possible to use the PDP-8/E to acquire 10,000 numbers in an on-line experiment, transmit the resulting data file from the PDP-8 disk to the IBM 370 system, verify the correctness of the transmitted data, and obtain the results of an analysis of variance (performed using a commercially available statistical package), all within 2 or $3 \mathrm{~h}$ from the start of the experimental session.

\section{REFERENCE NOTE}

1. Lemkin, P. Talk-10: A PDP-8/PDP-10 utility loader. Available from the Digital Equipment Corporation Users Society Library, DECUS No. 8-449C.

\section{REFERENCES}

Grupsmith, E. Linking large and small computers. Behavior Research Methods \& Instrumentation, 1978, 10, 269. 272.

Hitrz, F. L. How to make your laboratory computer into a smart timesharing terminal. Proceedings of the Spring 1975 DECUS Symposium. Maynard, Mass: Digital Equipment Corporation, 1975. Pp. 129-134.

\section{NOTES}

1. The situation is improving for laboratory users who can afford to keep up with the states of the art: FORTRAN IV, up to $128 \mathrm{~K}$ memory, floating-point hardware, etc. are becoming more available, at lower cost, for minicomputers. There is an intangible advantage, however, for the academic researcher in using the computer center rather than developing his or her own system hardware, that is, the availability of specialist consultants and access to expensive commercial statistical packages that are beyond the means of the individual laboratory.

2. The author has recently developed an expanded version of the program described here which records the log of all modem transmissions on the OS- 8 system mass storage device rather than printing it on the DECwriter and which supports the timesharing protocol of the PDP-10 as well as the IBM 370. Thus, two-way file exchange with the system files of both timesharing hosts is possible.

3. Normally, the connection between a computer and its console Teletype or other terminal is made by a $20-\mathrm{mA}$ current loop. In full duplex mode, there are two such loop connections (transmit and receive), with the current being supplied at the computer end of the circuit for each. The Teletype or terminal is, then, passive with respect to the current in the loop. In the normal timesharing system using an acoustic coupling to the telephone line, the coupling modem is, similarly, active and the terminal connected to it passive. Thus, the computer and modem may not be directly connected together since both are active devices (supply current). Fortunately, it is not difficult to make a mating connector using optically coupled isolators so that two active devices may be connected. The coupler appears to each active device to be a passive device. Diagrams for the mating connector may be obtained from the author.

4. Total cost of implementing the UART and modem was about $\$ 500$. A logic diagram of the UART interface for the PDP-8 is available from the author. Any 300-baud interface may be used for this application, however. The utility program described here will be submitted to the DECUS Library. Pending availability there, copies of the PAL 8 assembly listing may be obtained from the author.

5. The Intercept Jr. microcomputer with 256 words of read-write memory is available from distributors (e.g., Schweber Electronics or Computer Warehouse Store, Boston, Massachusetts) for about $\$ 280$. Up to three additional modules may be plugged into the basic board. A $1 \mathrm{~K}$ semiconductor memory (\$145), 20-mA loop or RS232 serial interface (\$85), and 12-bit parallel TTL-compatible I/O (\$125) are available. 